\title{
Novel Volumetric Compression Relaxation Testing of Skeletal Muscles
}

Anurag J. Vaidya, Department of Biomedical Engineering, Bucknell University, PA

Benjamin B. Wheatley, Department of Mechanical Engineering, Bucknell University, PA

Introduction: Computational models of the human body - such as those that simulate automotive impact - rely on accurate material properties for bodily tissues. However, the compressive behavior of skeletal muscle is not fully understood, particularly with regards to compression under confinement by surrounding tissue [1]. For example, it is likely that in vivo muscle experiences a variation between confined and unconfined volumetric boundary conditions, but nearly all previous studies have focused on muscle in unconfined compression (UC) or fully confined compression (CC) $[1,2,3]$. Thus, we have developed novel instrumentation to investigate the effects of volumetric boundary conditions (SC and $\mathrm{CC}$ ) on stress relaxation of skeletal muscles.

Materials and Methods: In semi-confinement (SC), cuboid samples are compressed in one direction, restricted in a second direction, and free of boundary conditions in the third orthogonal direction. In fully confined conditions (CC), samples are compressed in one direction and restricted in the other two orthogonal directions. Transverse oriented cuboids (average length $=24.9 \pm 1.1 \mathrm{~mm}$ for CC and $26.4 \pm 1.7 \mathrm{~mm}$ for SC, height $=8.89 \mathrm{~mm}$ for CC and $\mathrm{SC}$ ) were dissected from three porcine Tibialis Anterior (TA). For both CC and SC, two testing configurations were employed: fast-compression ( $\dot{\varepsilon}=15 \% / s)$ and slow-compression $(\dot{\varepsilon}=1 \% / s)$ to $15 \%$ strain with $\mathrm{n}=3$ for both SC and CC. All samples were subject to a 300s relaxation after compression. Data was collected by a $50 \mathrm{lbf}(222 \mathrm{~N})$ load. First Piola-Kirchhoff stress, peak stress, and nominal strain were determined post-hoc. A three term Prony series linear viscoelastic model was fit to normalized hold phase stress from all groups. A stochastic Monte-Carlo optimization identified initial guesses by minimizing percent error, then, a deterministic optimization performed finer fitting to experimental data. Normalized root mean square error was calculated to evaluate model fits.
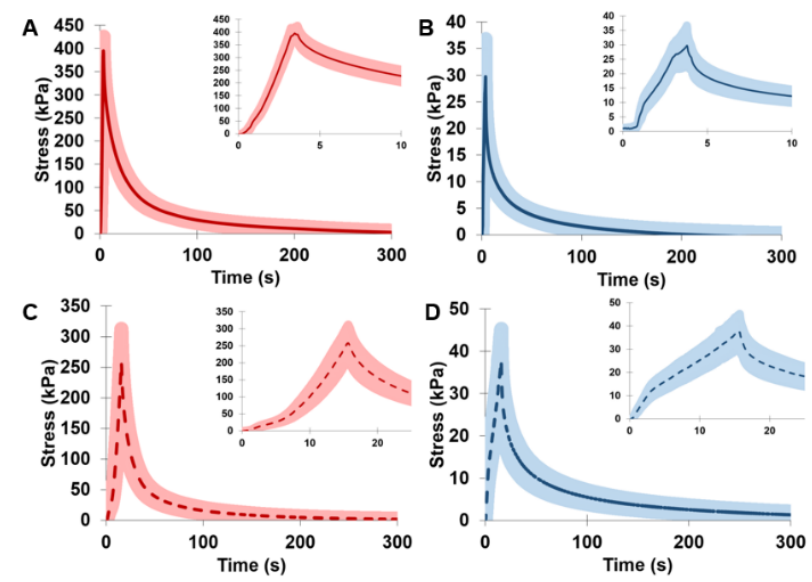

Figure 1: Stress-time curves with standard error for A) CC Fast B) SC Fast C) CC Slow D) SC

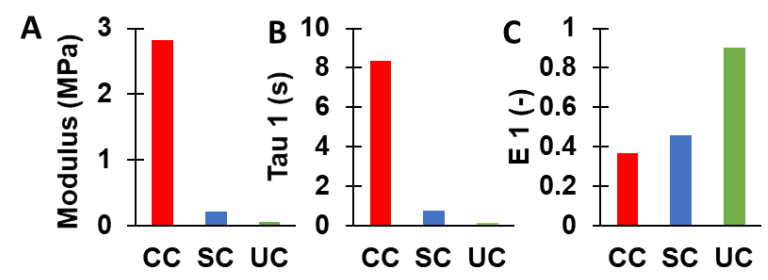

Figure 2: (A) Modulus (B) E1 parameter (C) Tau1 parameter for CC (red), SC (blue), and UC (green).
Results and Discussion: Figure 1 shows that despite the same strain levels, muscle in CC exhibited significantly higher peak stiffness than muscle in $\mathrm{SC}$ ( $\mathrm{p}$-value<0.001). In $\mathrm{CC}$, muscle stiffness increases with strain rate (p-value $=$ 0.02 . However, muscle's peak stress was not significantly different with strain rate for $\mathrm{SC}(\mathrm{p}$-value $=0.29)$. In a previous study, we determined that the peak modulus for muscle in unconfined compression was $52 \mathrm{kPa}$ [3]. The current study suggests the peak modulus for $\mathrm{SC}$ fast is $216 \mathrm{kPa}$ and that for $\mathrm{CC}$ fast is $2820 \mathrm{kPa}$. Moreover, it was previously shown that muscle in UC exhibited an $E_{1}$ parameter value of 0.90 and a $\tau_{1}$ value of $0.132 \mathrm{~s}$ [3]. $E_{1}$ and $\tau_{1}$ for SC found in this study fall between those for UC and CC. These results suggest that as muscle transitions from unconfined to confined compression, it becomes stiffer and relaxes less at a slower rate. Previously found $E_{1}$ for CC (0.6) is greater than current $E_{1}(0.37)$ and previous $\tau_{1}(0.33 \mathrm{~s})$ is smaller than current $\tau_{1}$ (8.35), which could be due to strain rate differences [3].

Conclusions: This study presents new data of passively compressed muscle under different volumetric boundary conditions (confined versus semiconfined). Since the parameters of viscoelastic model fit to semiconfined fall between those of confined and unconfined compression, we conclude that the study successfully presents data on a loading condition (semiconfined) that is somewhere in between fully confined and unconfined compression. Future work to investigate the role of saturating fluid and the development of a unified finite element model to capture all these conditions concurrently would be a significant contribution to the field.

References: [1] Moh., M., J Mech Behav Biomed Mater 62 (2016): 468-480 [2] Van Loocke, M., Jour. Biomech. 41.7 (2008): 1555-1566 [3] Vaidya, A., JMBBM 102 (2020): 103526. 